\title{
A C. elegans stretch receptor neuron revealed by a mechanosensitive TRP channel homologue
}

\author{
Wei $\mathrm{Li}^{1}$, Zhaoyang Feng ${ }^{1}$, Paul W. Sternberg ${ }^{3} \&$ X. Z. Shawn $\mathrm{Xu}^{1,2}$
}

The nematode Caenorhabditis elegans is commonly used as a genetic model organism for dissecting integration of the sensory and motor systems ${ }^{1}$. Despite extensive genetic and behavioural analyses that have led to the identification of many genes and neural circuits involved in regulating $C$. elegans locomotion behaviour ${ }^{1}$, it remains unclear whether and how somatosensory feedback modulates motor output during locomotion. In particular, no stretch receptors have been identified in C. elegans, raising the issue of whether stretch-receptor-mediated proprioception is used by $C$. elegans to regulate its locomotion behaviour. Here we have characterized TRP-4, the C. elegans homologue of the mechanosensitive TRPN channel. We show that trp-4 mutant worms bend their body abnormally, exhibiting a body posture distinct from that of wild-type worms during locomotion, suggesting that TRP-4 is involved in stretch-receptor-mediated proprioception. We show that TRP-4 acts in a single neuron, DVA, to mediate its function in proprioception, and that the activity of DVA can be stimulated by body stretch. DVA both positively and negatively modulates locomotion, providing a unique mechanism whereby a single neuron can fine-tune motor activity. Thus, DVA represents a stretch receptor neuron that regulates sensory-motor integration during C. elegans locomotion.

Transient receptor potential (TRP) proteins represent a superfamily of cation channels that are conserved from worms to humans and comprise seven subfamilies (TRPC, TRPV, TRPM, TRPN, TRPA, TRPP and TRPML) $)^{2}$ TRP channels have been implicated in various physiological processes ranging from fertilization to mechanosensation $^{2,3}$. We are particularly interested in TRP-4, a TRPN channel, because of its potential role in regulating mechanosensation (see below). We isolated two deletion mutants of TRP-4. Both $\operatorname{trp}-4$ alleles lack the regions encoding transmembrane domains and are likely to be null (Fig. 1a). TRP-4 has $\sim 40 \%$ sequence identity to and shares similar domain structures with zebrafish TRPN1 and Drosophila NOMPC, which encode putative mechanosensitive channels required for detecting sound vibration by hair cells in zebrafish and for sensing bristle displacement in flies, respectively ${ }^{4,5}$.

During locomotion, worms bend their body periodically, propagating a sinusoidal wave along their body axis ${ }^{1,6}$. We used an automated worm tracking system ${ }^{7}$ to record worm locomotion (see Methods), as conventional methods (human description) cannot provide quantitative measurement. Digitized images were then processed and subjected to data analysis. To facilitate data processing, we divided the worm body into 12 segments such that various locomotion parameters could be readily calculated, including frequency of body bending, extent of body bending (bending angles), track amplitude and track wavelength ${ }^{7}$ (Fig. 1b).

Both $\operatorname{trp}-4$ alleles showed two distinct locomotion defects. $\operatorname{trp}-4$ worms bent their body more frequently (Fig. 1e), a phenotype that we named 'fast bending'. As a result, the centroid speed in the mutant worms was increased (Fig. 1g). In addition, these mutant worms bent their body more deeply and showed a body posture distinct from that of wild-type worms during locomotion (Fig. 1c, d, f), suggesting a defect in stretch-receptor-mediated proprioception. This observation is consistent with the role of TRPN channels in mechanosensation, because proprioception is mediated by mechanosensitive channels ${ }^{4,5,8}$. As a result of this locomotion defect, mutant worms left behind deeper sinusoidal tracks than did wild-type worms (Fig. 1c, d). We named this second $\operatorname{trp}$-4 phenotype the 'exaggerated bending' phenotype. We used the extent of body bending (bending angles) to describe the exaggerated bending phenotype, because this parameter alone readily quantifies the curvature of the worm body independently of the body length. Neither the track amplitude nor the track wavelength alone is sufficient to do so. Similar methods are used in the clinic to diagnose scoliosis ${ }^{9}$. Both $\operatorname{trp}-4$ defects were
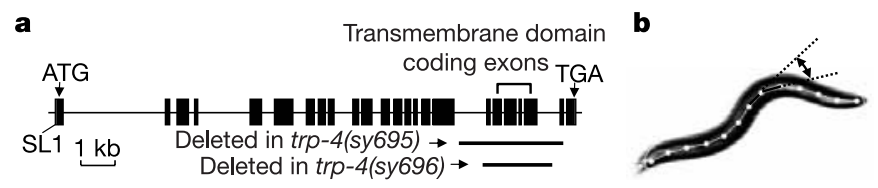

c d
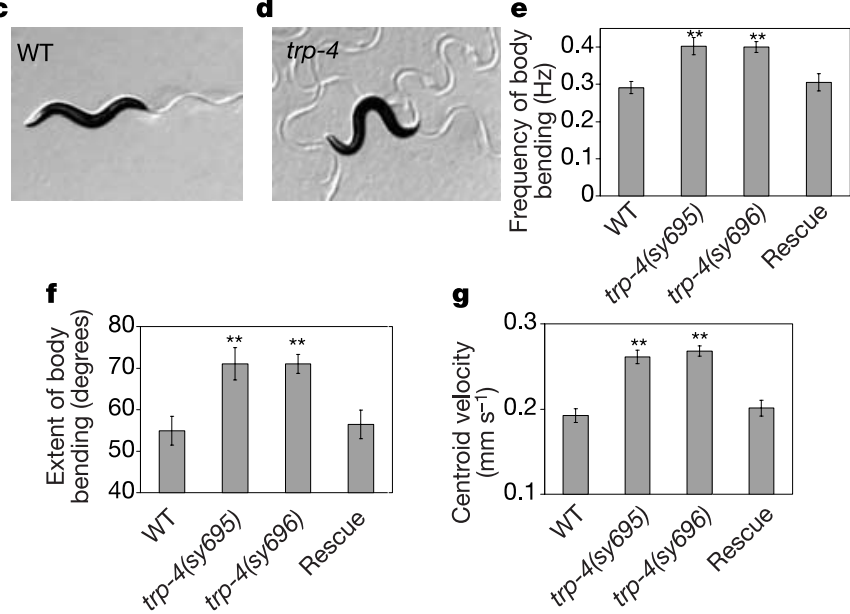

Figure 1 | Defective locomotion in trp-4 mutants. a, trp-4 gene structure and $\operatorname{trp}-4$ mutations. $\mathbf{b}$, Representative image showing that the worm body is divided into 12 segments (adapted from ref. 7). The arrow between the two dotted lines denotes the extent of body bending (bending angle) between the two segments. c, d, Snapshot images of a moving wild-type worm (c) and a trp-4(sy695) worm with abnormal body posture (d). e, Increased frequency of body bending in trp-4 mutants. f, $\operatorname{trp}-4$ mutant worms bend their body more deeply. $\mathrm{g}$, Centroid velocity is increased in $\operatorname{trp}-4$ mutants. Rescue indicates $\operatorname{trp}-4(s y 695)$ mutants expressing $\operatorname{Ex}[\operatorname{trp}-4:: y f p]$. Error bars indicate s.e.m. $(n \geq 12)$. ${ }^{\star *} P<0.005$. 
rescued by a transgene encoding full-length TRP-4 fused to yellow fluorescent protein (Fig. 1e-g).

trp- 4 has been reported to be expressed in the CEP and ADE dopamine neurons and in two interneurons, DVA and DVC 5 . We also observed TRP-4 expression in the PDE dopamine neurons, probably owing to our use of a longer promoter region $(\sim 7.5 \mathrm{~kb})$ of the $\operatorname{trp}-4$ gene. TRP-4 was highly enriched in the cilia of the dopamine neurons (Supplementary Fig. S1a), and was localized throughout the whole axon in DVA and DVC 5 (Supplementary Fig. S1b).

C. elegans dopamine neurons are sensory neurons with a morphology analogous to that of vertebrate hair cells in the inner ear (Supplementary Fig. S1c). Unlike the typical touch receptor neurons that detect gentle touch ${ }^{10,11}$, these dopamine neurons are mechanosensory neurons that sense mechanical attributes imposed by the surface material on which worms navigate ${ }^{12}$. As a result, worms slow down their frequency of body bending after encountering bacteria, a phenomenon called the 'basal slowing response"12. In the absence of bacteria, the frequency of body bending in trp-4 mutant worms was no longer faster than that in wild-type worms, suggesting that the fast bending phenotype of $\operatorname{trp}-4$ mutants might be due to a defect in the basal slowing response (Fig. 2a). Consequently, trp-4 mutant worms might always be in the highest state of locomotion, which in wildtype worms occurs only in the absence of bacteria ${ }^{12}$. In support of this idea, the dopamine-deficient mutant cat-2(e1112) (ref. 13) showed the same fast bending phenotype as the trp-4 mutant worms (Fig. 2a). In addition, expression of wild-type copies of $\operatorname{trp}-4$ specifically in dopamine neurons rescued the fast bending phenotype in $\operatorname{trp}-4$ mutants (Fig. 2a). Thus, dopamine neurons seem to mediate the fast bending phenotype in trp-4 mutants.

The extent of body bending in wild-type and trp-4 mutant worms was not affected by the presence of bacteria (Fig. 2b), however, suggesting that dopamine neurons are unlikely to mediate the exaggerated bending phenotype. Consistent with this hypothesis, the extent of body bending in the dopamine-deficient mutant cat-2 was similar to that of wild type (data not shown). We therefore examined the involvement of DVA and DVC, which also express TRP-4. These two neurons have their somata situated in the worm tail, and their axons span nearly the whole length of the worm body (Fig. 3a and Supplementary Fig. S1b). As TRP-4 is localized throughout the axons of DVA and DVC (Supplementary Fig. S1b), we speculated that when a worm bends its body, it stretches the plasma membrane of the axons of DVA and DVC. This stretch may then lead to activation of TRP-4, a mechanosensitive channel homologue, and consequently stimulate DVA and DVC (Fig. 3a). These two neurons would then signal negatively to the downstream command interneurons and ventral cord motor neurons onto which they primarily

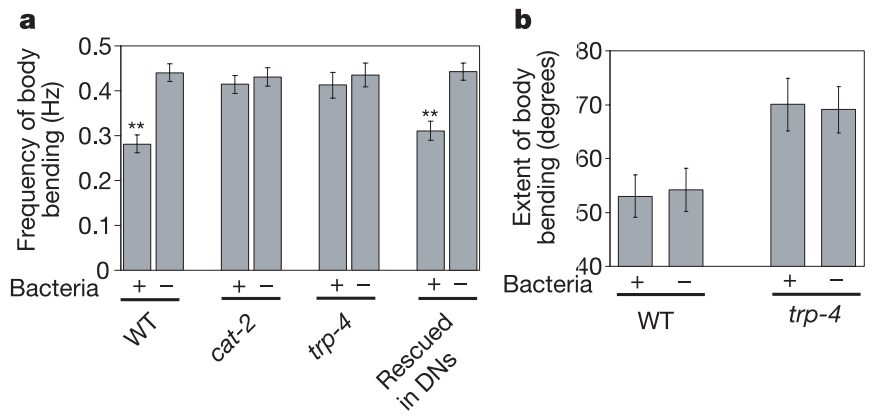

Figure 2 | Dopamine neurons mediate the fast bending phenotype of trp-4 mutants. a, The fast bending phenotype of $\operatorname{trp}-4$ mutants is mediated by dopamine neurons. Worms were tracked on plates with or without bacteria. DNs, dopamine neurons. Rescue indicates trp-4(sy695) mutants expressing Ex[Pdat-1::trp-4]. b, Extent of body bending is not affected by the presence of bacteria in wild-type or trp-4(sy695) worms. Data were processed from the same samples as in a. Error bars indicate s.e.m. $(n \geq 10)$. ${ }^{\star *} P<0.005$. synapse $^{14}$, inhibiting body-wall muscle contraction and thereby preventing exaggerated body bending. The command interneurons and ventral cord motor neurons are the two key components in the locomotion circuitry ${ }^{6}$. This model predicts that laser ablation of DVA and DVC in wild-type worms should mimic the exaggerated body bending seen in trp-4 mutant worms. Instead of augmenting the extent of body bending, however, laser ablation of DVA and DVC in wild-type worms slightly reduced the extent of body bending (Fig. 3e). Although the above proposed model seemed to be incorrect, this observation showed that DVA and DVC are important in regulating the extent of body bending.

Because killing of DVA and DVC led to a reduction in the extent of body bending, we considered that there might be a positive regulator of the extent of body bending in these two neurons in addition to the negative regulator TRP-4 (Fig. 3b). If so, laser ablation of DVA and DVC in wild-type worms would not be expected to recapitulate the trp-4 phenotype, because such ablation would eliminate both negative and positive regulators. This second model would also explain the exaggerated bending phenotype of $\operatorname{tr} p-4$ mutants, because loss of the negative regulator TRP-4 would unmask the effect of the putative positive regulator. If this second model is correct, laser ablation of DVA and DVC in the trp-4 mutant background should abrogate the activity of the remaining positive regulator, and should hence suppress the $\operatorname{trp}-4$ phenotype. Indeed, laser ablation of the DVA neuron alone was sufficient to suppress the exaggerated body bending phenotype in trp-4 mutant worms (Fig. 3c-e), whereas killing of DVC did not result in a significant effect (Fig. 3e). Expression of wildtype copies of TRP-4 specifically in DVA was also sufficient to rescue the exaggerated bending phenotype (Fig. 3e). These results suggest that the exaggerated bending phenotype of trp-4 mutants is mediated by the DVA neuron. Given that body bending periodically exerts local stretch on the plasma membrane of the DVA axon (Fig. 3a), our data suggest that DVA might function as a stretch-sensitive neuron.

To provide physiological evidence that the DVA neuron is stretchsensitive, we engineered a transgenic line expressing the genetically encoded $\mathrm{Ca}^{2+}$ sensor G-CaMP in the DVA neuron. DsRed2 was coexpressed with G-CaMP in DVA as an internal reference marker. G-CaMP has been successfully used as a non-invasive $\mathrm{Ca}^{2+}$ sensor in C. elegans and Drosophila neurons ${ }^{15,16}$. We first immobilized the DVA
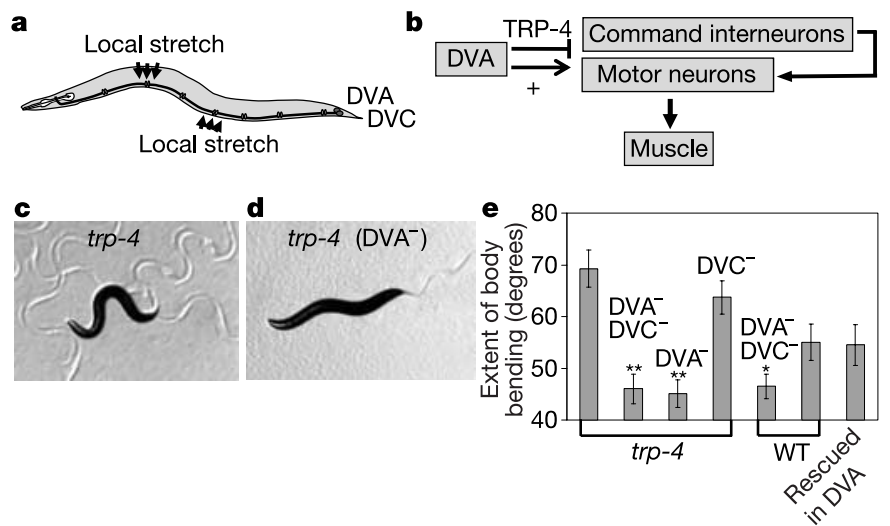

Figure 3 | TRP-4 functions in DVA to regulate the extent of body bending. a, Model showing that body bending locally stretches the plasma membrane of the DVA axon, potentially activating TRP-4 channels. Ovals depict TRP-4; arrows indicate the local stretch resulting from body bending. b, Second model proposing that the negative regulator TRP-4 acts together with an unknown positive factor in DVA to modulate the extent of muscle contraction. c, d, Snapshot images of a moving $\operatorname{trp}-4(s y 695)$ worm (c; duplicate of Fig. 1d) and a moving DVA-ablated $\operatorname{trp}-4$ (sy695) worm (d), showing that the exaggerated bending phenotype has been suppressed. e, DVA mediates the exaggerated bending phenotype in trp-4 mutants. Rescue indicates $\operatorname{trp}-4$ (sy695) mutants expressing Ex[Ptwk-16(DVA)::trp-4]. Error bars indicate s.e.m. $(n \geq 8) .{ }^{\star} P<0.005 ;{ }^{\star} P<0.05$. 
soma by gluing the tail of the worm on an agarose pad, while leaving the rest of the body free to move. Under these conditions, worms usually showed little movement, and no or little change in $\mathrm{Ca}^{2+}$ concentration was observed in DVA (Fig. 4b). On application of solution to the pad, worms began to bend their body in the liquid, triggering a robust increase in $\mathrm{Ca}^{2+}$ level in DVA $(n=29 / 29$; Fig. $4 \mathrm{a}-\mathrm{c}, \mathrm{e})$. We often observed repetitive $\mathrm{Ca}^{2+}$ spikes, with each spike presumably representing a body bending event (Fig. 4b). For those worms that vigorously bent their body at high frequency $\left(>3 \mathrm{~Hz}\right.$ ), no sharp $\mathrm{Ca}^{2+}$ spikes were observed (Fig. $4 \mathrm{c}$ ), probably owing to the relatively slow dissociation kinetics of G-CaMP (half time $=200 \mathrm{~ms})^{17}$. We did not detect a significant $\mathrm{Ca}^{2+}$ response in $\operatorname{trp}-4$ mutant worms under the same conditions $(n=0 / 16$; Fig. $4 \mathrm{~d}$, e), apart from a very brief $\mathrm{Ca}^{2+}$ transient that was often observed at the onset of liquid application $(n=8 / 16$; Fig. $4 d)$. Such a deficit in $\mathrm{Ca}^{2+}$ response was unlikely to be due to a defect in DVA excitability, because DVA isolated from $\operatorname{trp}-4$ mutant embryos retained the ability to respond to membrane depolarization induced by potassium chloride (Supplementary Fig. S3). These data suggest that body stretch is sufficient to stimulate TRP-4-dependent activity in DVA.

As DVA receives synaptic input from other mechanosensory neurons including PDE, PLM and PVD, we carried out similar imaging experiments on PDE-ablated wild-type worms and mec-3 mutant (lacking PLM and PVD) worms ${ }^{18,19}$, and observed similar $\mathrm{Ca}^{2+}$ responses (Fig. 4e and Supplementary Fig. S2c-f). No significant difference was detected in the extent of body bending between these worms and wild-type worms (Fig. 4f). We also observed body-bending-evoked $\mathrm{Ca}^{2+}$ transients in unc-13 worms, in which synaptic transmission is essentially eliminated ${ }^{20}$ (Fig. 4e and Supplementary Fig. S2g, h). Thus, DVA seems to be the primary neuron mediating the body-bending-evoked $\mathrm{Ca}^{2+}$ transients.

To obtain further evidence that DVA is stretch-sensitive, we immobilized the worm's tail with glue, held its nose tip with a glass pipette, and then manually bent its body (Fig. 4g). Bending the worm's body evoked a sustained increase in $\mathrm{Ca}^{2+}$ in the DVA neuron, which decayed to basal levels after cessation of the stimulus $(n=11 / 12$; Fig. $4 \mathrm{~h})$. The amplitude of the response seemed to be graded (Supplementary Fig. S4), and no sustained response was observed until the bending angle reached a specific threshold $\left(\sim 50^{\circ}\right)$, consistent with a role for TRP-4 in antagonizing overcontraction of body-wall muscles (Fig. 4h and Supplementary Fig. S4). No such response was detected in trp-4 mutant worms $(n=0 / 11)$, although a very brief $\mathrm{Ca}^{2+}$ transient was observed $(n=11$; Fig. $4 \mathrm{i}, \mathrm{j})$. These observations indicate that DVA may be stretch-sensitive.

In summary, we have presented evidence supporting the notion that DVA is a stretch receptor neuron. Our results indicate that stretch-receptor-mediated proprioception is important for proper motor function in C. elegans. Nevertheless, our study does not exclude the presence of additional stretch receptors in C. elegans, for example, the undifferentiated processes of ventral cord motor neurons ${ }^{6}$. We have also shown the presence of a putative positive regulator in DVA; however, its activity might not be primarily mediated by $\mathrm{Ca}^{2+}$. We propose that such a positive regulator may function to promote the extent of muscle contraction initially, and
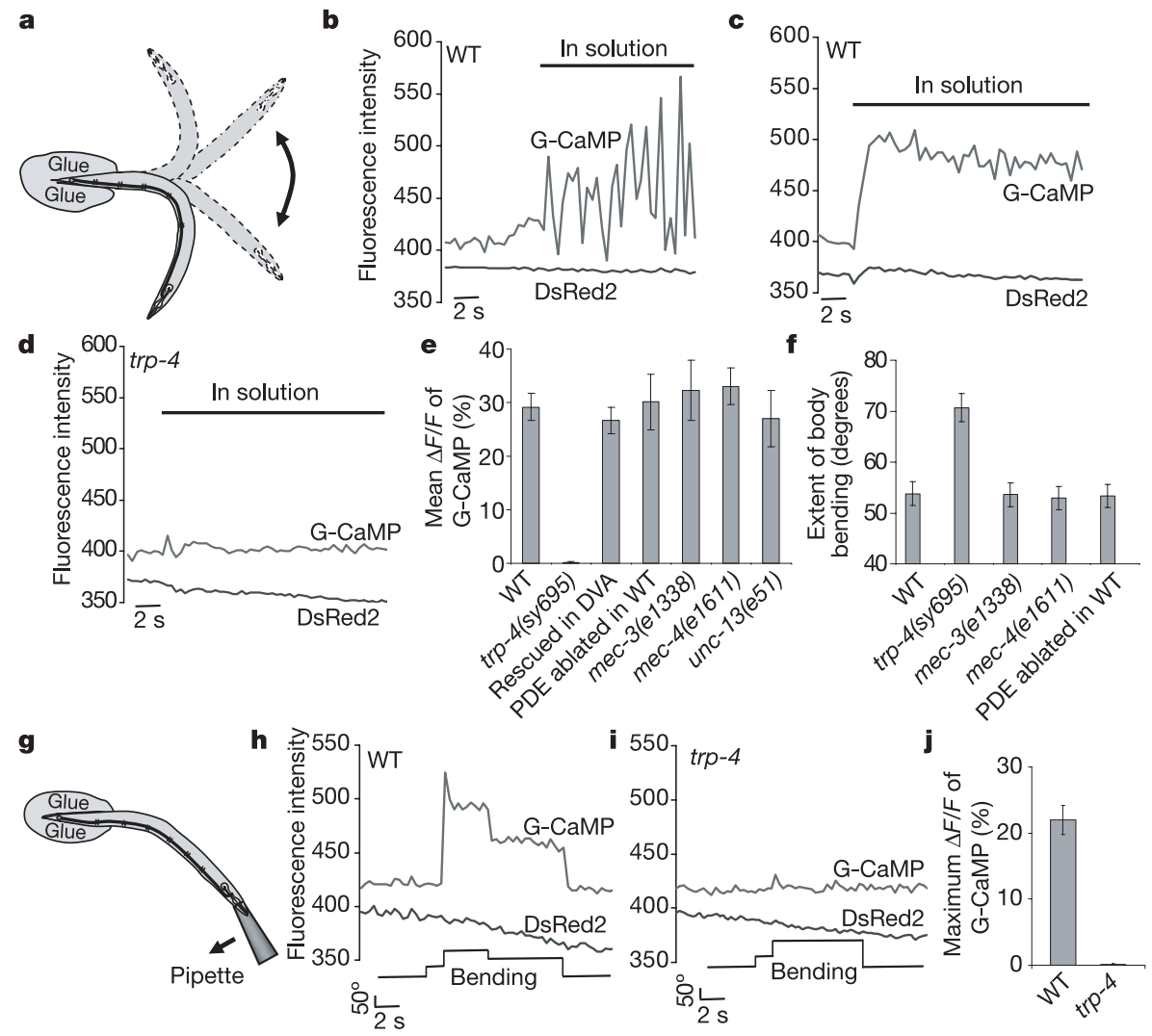

Figure 4 The DVA neuron is stretch-sensitive. a, A worm, with its tail glued, freely bends its body. b, c, Body-bending-evoked $\mathrm{Ca}^{2+}$ signals in DVA. d, Body bending does not induce a significant $\mathrm{Ca}^{2+}$ response in $\operatorname{trp}-4(s y 695)$. e, Mean G-CaMP fluorescence change in DVA. The mean peak fluorescence of G-CaMP in the first $20 \mathrm{~s}$ after liquid application was measured. Rescued in DVA indicates trp-4(sy695) mutants expressing Ex[Ptwk-16(DVA)::trp-4]. f, Lack of PDE or gentle- and harsh-touch receptor neurons does not have a significant effect on the extent of body bending. $g$, Manual bending of the body of a glued worm by a glass pipette. $\mathbf{h}$, i, Manual bending of a worm's body induces an increase in $\mathrm{Ca}^{2+}$ in DVA of wild-type (h), but not trp-4(sy695) mutant (i) worms. Representative traces are shown. j, Maximal increase in G-CaMP fluorescence in DVA. Wild-type and mutant worms were bent to $\sim 120^{\circ}$, at which point the $\mathrm{Ca}^{2+}$ concentration in DVA reached its maximum (Supplementary Fig. S5). The fluorescence intensity of DsRed2 slowly decreased because of its relatively fast bleach as compared with G-CaMP. Error bars indicate s.e.m. $(n \geq 5)$. 
that when muscle contraction reaches a specific extent, the negative regulator TRP-4 then signals to prevent further contraction of muscle cells. This dual control of the extent of body bending by DVA would confer on worms the capacity to tune the extent of body-wall muscle contraction, providing a unique mechanism for the general fine control of motor activity by proprioceptive stretch receptors.

Among the best-characterized stretch receptors in vertebrates are muscle spindles and Golgi tendon organs, whereas chordotonal organs represent the best-analysed proprioceptors in arthropods $s^{8,21}$. In humans, muscle spindles signal to promote muscle contraction, and Golgi tendon organs function to repress muscle contraction to avoid muscle damage ${ }^{8}$. Such antagonistic roles of vertebrate muscle spindles and Golgi tendon organs seem analogous to those of the DVA neuron in C. elegans, except that DVA, as a single cell, seems to carry out both functions. Thus, the phenomenon that proprioceptormediated somatosensory feedback can both positively and negatively modulate muscle activity seems to be present in both organisms. We propose that some of the basic principles underlying somatosensory feedback regulation of motor output are evolutionarily conserved.

\section{METHODS}

Behavioural analysis and the worm tracker. L4 hermaphrodites were picked $16 \mathrm{~h}$ before behavioural analysis. Worms were tracked for $5 \mathrm{~min}$ at $20^{\circ}$ on NGM plates spread with a thin layer of freshly grown OP50 bacteria as described ${ }^{7}$. For tracking in the absence of bacteria, the supernatant of OP50 culture was spread on tracking plates. The tracking system consists of a stereomicroscope mounted with a Cohu 7800 digital camera, a digital motion system (Parker Automation) that follows worm movement, and laboratory-developed software. The vision/ motion data were compressed and integrated into AVI format for feature extraction. To quantify the extent of body bending (bending angles), binarized worm images were thinned to obtain the 'skeleton image' of the worm and broken into 12 equal-length segments with real world coordinates. For simplicity and consistency, we selected the middle segments (segments 6 and 7) of the worm body for quantification unless otherwise specified. Our wild-type and cat-2 data are quantitatively similar to reported data ${ }^{12}$, although our frequency of body bending is a little slower $(\sim 20 \%)$, probably because we averaged data from the whole tracking period $(5 \mathrm{~min})$. The centroid velocity was calculated by a described method ${ }^{7}$, which measures the speed of wave propagation but not the vector speed during locomotion.

Molecular biology. The $5^{\prime}$ and $3^{\prime}$ ends of the $\operatorname{trp}-4$ coding regions (Fig. 1) were determined by rapid amplification of cloned ends. We used the same G-CaMP transgene for all $\mathrm{Ca}^{2+}$ imaging studies by crossing it into different genetic backgrounds. See Supplementary Information for details.

$\mathrm{Ca}^{2+}$ imaging. $\mathrm{Ca}^{2+}$ imaging was done on an Axiovert 200 microscope (Zeiss) under a $\times 40$ objective. Images were acquired with a CoolSnap CCD camera (Roper) and processed by Ratiotool software (ISeeimaging). See Supplementary Information for details.

\section{Received 7 July; accepted 19 December 2005}

1. Samuel, A. D. \& Sengupta, P. Sensorimotor integration: locating locomotion in neural circuits. Curr. Biol. 15, R341-R343 (2005)

2. Montell, C. The TRP superfamily of cation channels. Sci STKE 2005, re3 (2005)
3. Xu, X. Z. S. \& Sternberg, P. W. A C. elegans sperm TRP protein required for sperm-egg interactions during fertilization. Cell 114, 285-297 (2003).

4. Sidi, S., Friedrich, R. W. \& Nicolson, T. NompC TRP channel required for vertebrate sensory hair cell mechanotransduction. Science 301, 96-99 (2003).

5. Walker, R. G., Willingham, A. T. \& Zuker, C. S. A Drosophila mechanosensory transduction channel. Science 287, 2229-2234 (2000).

6. Chalfie, M. \& White, J. in The Nematode Caenorhabditis elegans (ed. Wood, W. B.) 337-391 (Cold Spring Harbor Laboratory Press, New York, 1988).

7. Cronin, C. J. et al. An automated system for measuring parameters of nematode sinusoidal movement. BMC Genet. 6, 5 (2005).

8. Hall, W. C. \& Nicolelis, M. in Neuroscience (eds Purves, D. et al.) 347-368 (Sinauer Associates, Sunderland, 2001).

9. Goldberg, C. J. et al. in Research Into Spinal Deformities (ed. Stokes, I. A. F.) 53-56 (International Research Society of Spinal Deformities, Amsterdam, 1999).

10. Ernstrom, G. G. \& Chalfie, M. Genetics of sensory mechanotransduction. Annu. Rev. Genet. 36, 411-453 (2002).

11. Goodman, M. B. \& Schwarz, E. M. Transducing touch in Caenorhabditis elegans. Annu. Rev. Physiol. 65, 429-452 (2003).

12. Sawin, E. R., Ranganathan, R. \& Horvitz, H. R. C. elegans locomotory rate is modulated by the environment through a dopaminergic pathway and by experience through a serotonergic pathway. Neuron 26, 619-631 (2000).

13. Lints, R. \& Emmons, S. W. Patterning of dopaminergic neurotransmitter identity among Caenorhabditis elegans ray sensory neurons by a TGF $\beta$ family signaling pathway and a Hox gene. Development 126, 5819-5831 (1999).

14. White, J. G., Southgate, E., Thomson, J. N. \& Brenner, S. The structure of the nervous system of the nematode Caenorhabditis elegans. Phil. Trans. R. Soc. Lond. B 314, 1-340 (1986).

15. Kahn-Kirby, A. H. et al. Specific polyunsaturated fatty acids drive TRPV-dependent sensory signaling in vivo. Cell 119, 889-900 (2004)

16. Wang, J. W., Wong, A. M., Flores, J., Vosshall, L. B. \& Axel, R. Two-photon calcium imaging reveals an odor-evoked map of activity in the fly brain. Cell $112,271-282$ (2003).

17. Nakai, J., Ohkura, M. \& Imoto, K. A high signal-to-noise $\mathrm{Ca}^{2+}$ probe composed of a single green fluorescent protein. Nature Biotechnol. 19, 137-141 (2001).

18. Way, J. C. \& Chalfie, M. The mec-3 gene of Caenorhabditis elegans requires its own product for maintained expression and is expressed in three neuronal cell types. Genes Dev. 3, 1823-1833 (1989).

19. Driscoll, M. \& Chalfie, M. The mec- 4 gene is a member of a family of Caenorhabditis elegans genes that can mutate to induce neuronal degeneration. Nature 349, 588-593 (1991).

20. Richmond, J. E., Davis, W. S. \& Jorgensen, E. M. UNC-13 is required for synaptic vesicle fusion in C. elegans. Nature Neurosci. 2, 959-964 (1999).

21. Cattaert, D. \& Le Ray, D. Adaptive motor control in crayfish. Prog. Neurobiol. $63,199-240$ (2001)

Supplementary Information is linked to the online version of the paper at www.nature.com/nature.

Acknowledgements We thank J. Nakai for the G-CaMP plasmid; B. Perry for help with deletion libraries; $\mathrm{C}$. Cronin for assistance with data analysis; G. Schindelman, N. Moghal, C. Montell and A. Ward for comments; and G. Medina, E. Larkspur and R. Mahapatra for technical assistance. Some strains were obtained from the Caenorhabditis Genetics Center. This work was supported by the University of Michigan BSSP Scholar Program (X.Z.S.X.), the Helen Hay Whitney Foundation (X.Z.S.X), and the Howard Hughes Medical Institute, of which P.W.S. is an investigator.

Author Information Reprints and permissions information is available at npg.nature.com/reprintsandpermissions. The authors declare no competing financial interests. Correspondence and requests for materials should be addressed to X.Z.S.X (shawnxu@umich.edu). 